\title{
Geometric Combination of Multiple Terrestrial Network Solutions
}

\author{
J. Y. $\operatorname{Han}^{1}$; B. H. W. van Gelder, M.ASCE'; T. Soler, M.ASCE${ }^{3}$; and R. A. Snay ${ }^{4}$
}

\begin{abstract}
Terrestrial network solutions prepared by different institutes and/or at different epochs imply different reference frame definitions, since various reference stations and processing strategies may be involved. A combination procedure, utilizing a time-variant similarity transformation model, enables a geometric integration of multiple solutions into a common reference frame definition. Additional benefits, including an elimination of systematic bias and a cross check on the quality of each individual network solution, could also be achieved. In this study, a combination approach which takes into account complete geometric interrelations between multiple solutions is developed. With the observable dependency analysis procedure, the proposed approach guarantees a self-consistent, more meaningful, combination solution regardless of the choice of a reference solution. Numerical tests have been performed on actual International Global Navigation Satellite System (GNNS) Service (IGS) and National Geodetic Survey (NGS) solutions using the Geodetic Network Analysis Tool software developed along with this study. Results reveal potential problems if the proposed analysis procedure is not implemented in a combination solution.
\end{abstract}

DOI: 10.1061/(ASCE)0733-9453(2008)134:4(126)

CE Database subject headings: Geodetic surveys; Satellites; Numerical analysis.

\section{Introduction}

Fostered by the developments of space technologies and satellite geodesy, precisely defined terrestrial reference frames have been established globally and locally everywhere throughout the world. Examples are the International Terrestrial Reference Frame (ITRF) (see, e.g., Boucher and Altamimi 1989, and Ray et al. 1999), the North American Datum 1983 (NAD83) (Schwarz 1989), the European Terrestrial Reference System 89 (ETRS89) (Boucher and Altamimi 1992), the Geocentric Datum of Australia 1994 (GDA94) (Steed 1995), etc. Additionally, the weekly solutions provided by the International Global Navigation Satellite System (GNSS) Service (IGS) can also be regarded as a global reference frame with more up-to-date information (Ferland 2004). These newly established reference frames are designated to provide a reliable basis for any spatial applications. However, due to the earth dynamics, points on the Earth surface are moving with respect to one another (see e.g., DeMets et al. 1990). As a result, any reference frame which is realized by the positional coordi-

\footnotetext{
${ }^{1}$ Assistant Professor, Dept. of Civil Engineering, National Taiwan Univ., Taipei 106, Taiwan (corresponding author). E-mail: jyhan@ntu. edu.tw

${ }^{2}$ Associate Professor, Land Surveying and Geomatics Engineering, School of Civil Engineering, Purdue Univ., West Lafayette, IN 47907.

${ }^{3}$ Chief Technical Officer, Spatial Reference Systems Division, National Geodetic Survey, NOS, National Oceanic and Atmospheric Administration, Silver Spring, MD 20910.

${ }^{4}$ Chief, Spatial Reference Systems Division, National Geodetic Survey, NOS, National Oceanic and Atmospheric Administration, Silver Spring, MD 20910.

Note. Discussion open until April 1, 2009. Separate discussions must be submitted for individual papers. The manuscript for this paper was submitted for review and possible publication on October 9, 2007; approved on March 14, 2008. This paper is part of the Journal of Surveying Engineering, Vol. 134, No. 4, November 1, 2008. CASCE, ISSN 0733-9453/2008/4-126-131/\$25.00.
}

nates and velocities of a selected set of reference sites also evolves with time.

In a classic approach, a terrestrial network solution is generated by constraining the positions/velocities of a specific set of reference sites. Those reference sites (also referred to as control or fiducial points) from different reference frames and/or at different epochs will result in solutions with different reference frame definitions. Furthermore, various data processing strategies and observational techniques may also produce solutions that have slightly different reference frame definitions. In order to make use of all the solutions that come with nonunified reference frame definitions, a combination approach can be applied. In a combination approach, all available solutions are homogeneously transformed to a common reference frame by postulating a geometric similarity between each pair of solutions. After estimating (several sets of) similarity transformation parameters and the transformed coordinates/velocities in a predefined reference frame, a combination solution containing all the information from different solutions but expressed in a common reference frame is thus generated (see, e.g., Altamimi et al. 2002; Altamimi and Boucher 2003; Ray and Altamimi 2005). A combination approach also provides an opportunity to perform a cross-check on the consistency between different solutions and to reestimate their accuracies under the combination model, using the variance estimation technique for heterogeneous data sets described in Grafarend (1984) and Sahin et al. (1992).

The major emphasis of this study is thus on the investigation of a combination approach that rigorously takes into account the complete geometric relationships between multiple network solutions. By identifying all possible independent interrelations between multiple solutions and implementing them in a combination procedure, the resulting combination solution is more self-consistent and comprehensive. As will be shown in the "Case Study" section, a significant problem could occur in the final so- 
lution if the combination approach described in this study is not implemented.

\section{Mathematical Model}

In order to align network solutions that are based on different reference frame definitions, a time-variant similarity transformation is typically applied (see e.g., Soler 1998; Snay 1999; Soler and Snay 2004; Boucher and Altamimi 2001; Altamimi et al. 2002; Han and van Gelder 2006). For multiple network solutions to be transformed to a common reference frame, one can write

$$
\overrightarrow{\mathbf{X}}_{\mathbf{C}}=\sigma_{\text {IC }}\left[\mathbf{R}_{\text {IC }}\right] \overrightarrow{\mathbf{X}}_{\mathbf{I}}+\overrightarrow{\mathbf{T}}_{\text {IC }}
$$

Here $\overrightarrow{\mathbf{X}}_{\mathbf{I}}=$ positional vector in the solution I (input solution); $\overrightarrow{\mathbf{X}}_{\mathbf{C}}=$ positional vector in the solution $\mathbf{C}$ (output/combination solution); and $\sigma_{\text {IC }},\left[\mathbf{R}_{\mathbf{I C}}\right]$, and $\overrightarrow{\mathbf{T}}_{\mathbf{I C}}=$ similarity transformation parameters (i.e., scale, rotations, and translations) from solution $\mathbf{I}$ to solution C. For transforming the velocity vectors from different solutions, the following equation is applied:

$$
\overrightarrow{\mathbf{V}}_{\text {C }}=\left[\dot{\sigma}_{I C}\left[\mathbf{R}_{I C}\right]+\sigma_{I C}\left[\dot{\mathbf{R}}_{I C}\right]\right] \overrightarrow{\mathbf{X}}_{I}+\sigma_{I C}\left[\mathbf{R}_{I C}\right] \overrightarrow{\mathbf{V}}_{I}+\dot{\mathbf{T}}_{I C}
$$

Here $\overrightarrow{\mathbf{V}}_{\mathbf{I}}$ =velocity vector in the solution I (input solution); $\overrightarrow{\mathbf{V}}_{\mathbf{C}}=$ velocity vector in the solution $\mathbf{C}$ (output/combination solution); and $\dot{\sigma}_{\mathbf{I C}},\left[\dot{\mathbf{R}}_{\mathbf{I C}}\right]$, and $\dot{\mathbf{T}}_{\mathbf{I C}}=$ time derivatives of the similarity transformation parameters, which represent the timedependent variations of the scale, rotational, and translational parameters from solution $\mathbf{I}$ to solution $\mathbf{C}$.

When multiple network solutions $(\mathbf{I}=1, \ldots, \mathbf{n})$ are given and to be combined, a reference solution should be assigned in order to provide partial constraints for the combination solution C. Consequently, the combination solution is based on the same reference frame definition as the chosen reference solution. Then, by estimating (multiple sets of) the similarity transformation parameters and their time-dependent variations $\left(\sigma_{\text {IC }},\left[\mathbf{R}_{\mathbf{I C}}\right], \overrightarrow{\mathbf{T}}_{\mathbf{I C}}, \dot{\sigma}_{\mathbf{I C}},\left[\dot{\mathbf{R}}_{\mathbf{I C}}\right], \overrightarrow{\mathbf{T}}_{\mathbf{I C}}\right)_{\mathbf{I}=1 \ldots . \mathbf{n}}$ in Eqs. (1) and (2), all the positions and velocities from each input solution can be transformed accordingly into a common reference frame. Fig. 1 illustrates this combination procedure. Note that the unique points in the figure represent the points that exist in only one of the solutions.

It should also be pointed out that a time-variant similarity transformation model is typically rank deficient. Fourteen parameters are presented in the model but only seven of them are independent. Consequently, it is not possible to obtain the estimates of all 14 parameters in one single step in the sense of the leastsquares estimation. Hence, a stepwise approach proposed by Han and van Gelder (2006) should be applied to avoid the rankdeficiency problem and to give more meaningful parameter estimates without deteriorating the rigorousness of the transformation model.

\section{Geometric Dependency between Multiple Similarity Transformations}

Since a geometric similarity is postulated between each pair of network solutions, all those relations should be identified and used in a combination for rigorousness. However, it can be proven that the transformations between multiple pairs of solu-

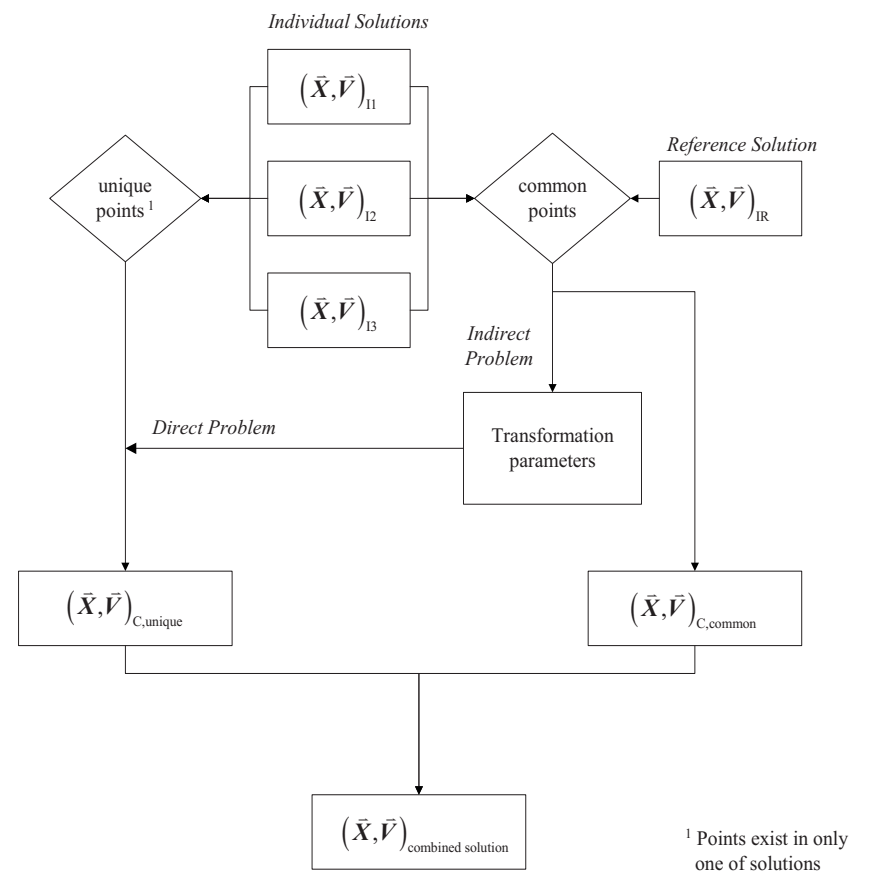

Fig. 1. Procedure for combining multiple network solutions

tions are not all independent. Here a three-solution case is used to illustrate this issue. Suppose the positional coordinates of three solutions to be combined are $\overrightarrow{\mathbf{X}}_{\mathbf{C}}, \overrightarrow{\mathbf{X}}_{1}$, and $\overrightarrow{\mathbf{X}}_{2}$. One can write (at most) three transformations between them

$$
\begin{aligned}
& \overrightarrow{\mathbf{X}}_{\mathbf{C}}=\sigma_{1 \mathbf{C}}\left[\mathbf{R}_{1 \mathbf{C}}\right] \overrightarrow{\mathbf{X}}_{1}+\overrightarrow{\mathbf{T}}_{1 \mathbf{C}} \\
& \overrightarrow{\mathbf{X}}_{\mathbf{C}}=\sigma_{2 \mathbf{C}}\left[\mathbf{R}_{2 \mathbf{C}}\right] \overrightarrow{\mathbf{X}}_{2}+\overrightarrow{\mathbf{T}}_{2 \mathbf{C}} \\
& \overrightarrow{\mathbf{X}}_{2}=\sigma_{12}\left[\mathbf{R}_{12}\right] \overrightarrow{\mathbf{X}}_{1}+\overrightarrow{\mathbf{T}}_{12}
\end{aligned}
$$

However, from Eqs. (3) and (4), it can be shown that

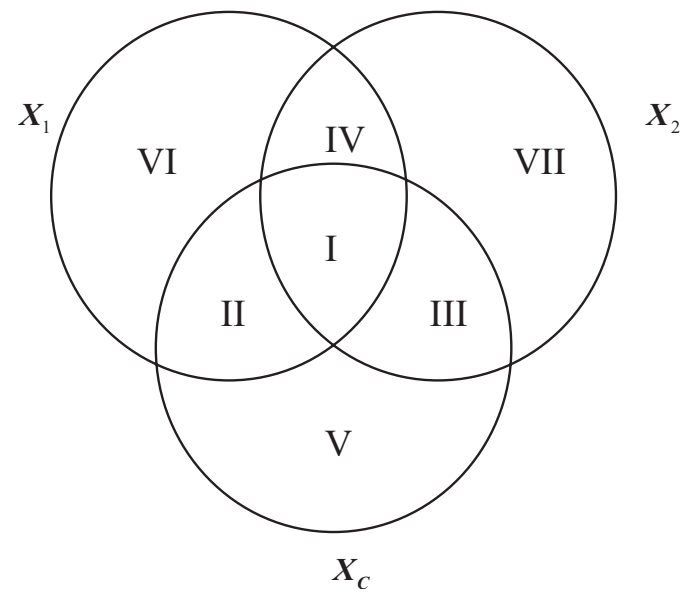

Fig. 2. Venn diagram showing duplication of information in determining transformation parameters for three-solution combination 


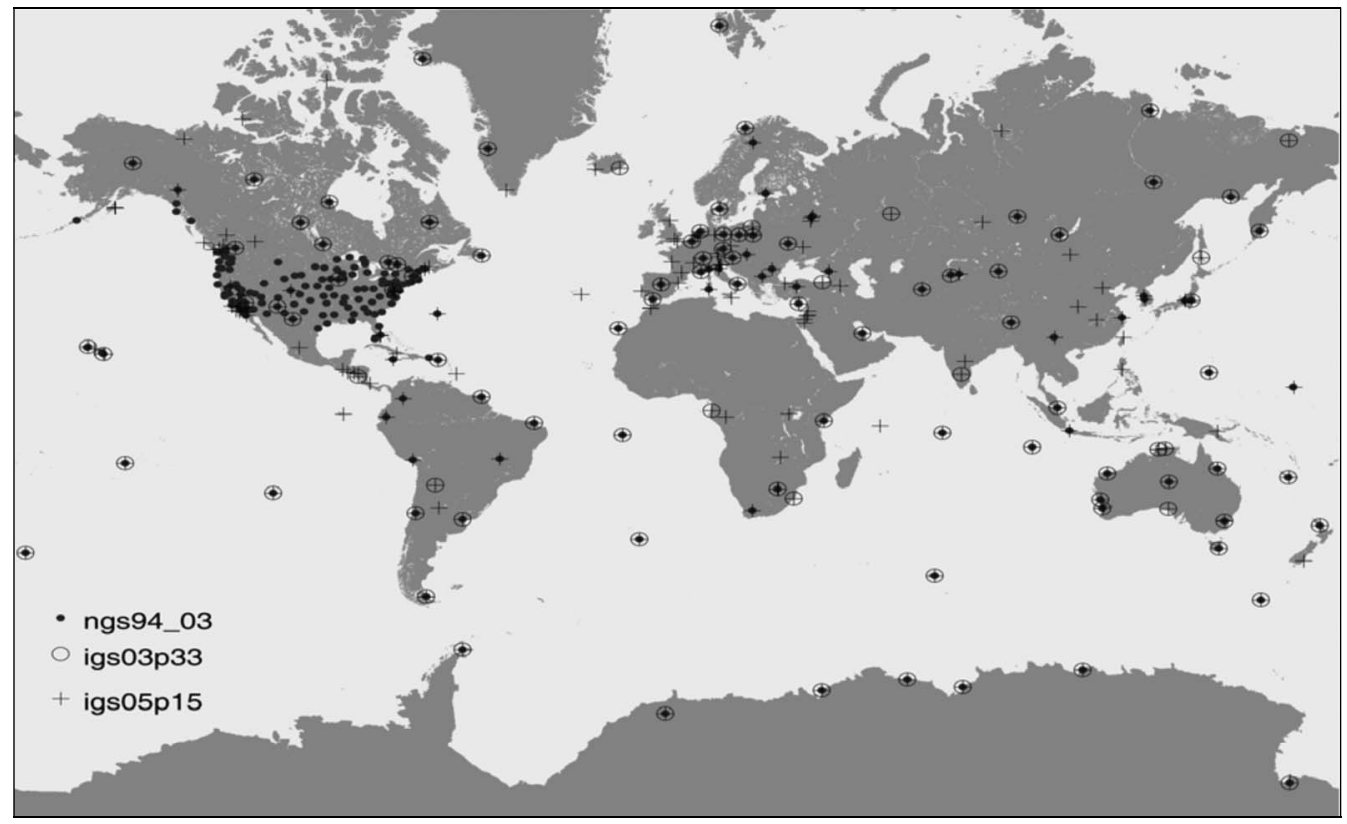

Fig. 3. Site distributions of three geodetic solutions in combination

$$
\overrightarrow{\mathbf{X}}_{2}=\frac{\sigma_{1 \mathbf{C}}}{\sigma_{2 \mathbf{C}}}\left[\mathbf{R}_{2 \mathbf{C}}\right]^{\mathbf{T}}\left[\mathbf{R}_{1 \mathbf{C}}\right] \overrightarrow{\mathbf{X}}_{1}+\frac{1}{\sigma_{2 \mathbf{C}}}\left[\mathbf{R}_{2 \mathbf{C}}\right]^{\mathbf{T}}\left(\overrightarrow{\mathbf{T}}_{1 \mathbf{C}}-\overrightarrow{\mathbf{T}}_{2 \mathbf{C}}\right)
$$

By comparing Eq. (5) with Eq. (6), one can immediately write

$$
\begin{gathered}
\sigma_{12}=\frac{\sigma_{1 \mathbf{C}}}{\sigma_{2 \mathbf{C}}} \\
{\left[\mathbf{R}_{12}\right]=\left[\mathbf{R}_{2 \mathbf{C}}\right]^{\mathbf{T}}\left[\mathbf{R}_{1 \mathbf{C}}\right]} \\
\overrightarrow{\mathbf{T}}_{12}=\frac{1}{\sigma_{2 \mathbf{C}}}\left[\mathbf{R}_{2 \mathbf{C}}\right]^{\mathbf{T}}\left(\overrightarrow{\mathbf{T}}_{1 \mathbf{C}}-\overrightarrow{\mathbf{T}}_{2 \mathbf{C}}\right)
\end{gathered}
$$

Eqs. (7)-(9) reveal the fact that the third set of transformation parameters $\left(\mathbf{P}_{12}\right)$ is dependent on the other two sets of parameters $\left(\mathbf{P}_{1 \mathbf{C}}\right.$ and $\left.\mathbf{P}_{2 \mathbf{C}}\right)$. Consequently, if one wants to fix the complete geometric relationships between these three solutions, one should use condition equations (3), (4), and (6) instead of Eqs. (3)-(5) for the reason just stated.

Following similar derivations, the equations to combine velocities from three solutions can also be written with two sets of independent transformation parameters

$$
\overrightarrow{\mathbf{V}}_{\mathbf{C}}=\left[\dot{\sigma}_{1 \mathbf{C}}\left[\mathbf{R}_{1 \mathbf{C}}\right]+\sigma_{1 \mathbf{C}}\left[\dot{\mathbf{R}}_{1 \mathbf{C}}\right]\right] \overrightarrow{\mathbf{X}}_{1}+\sigma_{1 \mathbf{C}}\left[\mathbf{R}_{1 \mathbf{C}}\right] \overrightarrow{\mathbf{V}}_{1}+\dot{\mathbf{T}}_{1 \mathbf{C}}
$$

$$
\overrightarrow{\mathbf{V}}_{\mathbf{C}}=\left[\dot{\sigma}_{2 \mathbf{C}}\left[\mathbf{R}_{2 \mathbf{C}}\right]+\sigma_{2 \mathbf{C}}\left[\dot{\mathbf{R}}_{2 \mathbf{C}}\right]\right] \overrightarrow{\mathbf{X}}_{2}+\sigma_{2 \mathbf{C}}\left[\mathbf{R}_{2 \mathbf{C}}\right] \overrightarrow{\mathbf{V}}_{2}+\dot{\overrightarrow{\mathbf{T}}}_{2 \mathbf{C}}
$$

$$
\begin{aligned}
\overrightarrow{\mathbf{V}}_{2}= & \frac{\left[\mathbf{R}_{2 \mathbf{C}}\right]^{\mathbf{T}}}{\sigma_{2 \mathbf{C}}}\left[\dot{\sigma}_{1 \mathbf{C}}\left[\mathbf{R}_{1 \mathbf{C}}\right]+\sigma_{1 \mathbf{C}}\left[\dot{\mathbf{R}}_{1 \mathbf{C}}\right]-\frac{\dot{\sigma}_{2 \mathbf{C}} \sigma_{1 \mathbf{C}}}{\sigma_{2 \mathbf{C}}}\left[\mathbf{R}_{1 \mathbf{C}}\right]-\sigma_{1 \mathbf{C}}\left[\dot{\mathbf{R}}_{2 \mathbf{C}}\right]\right. \\
& \left.\times\left[\mathbf{R}_{2 \mathbf{C}}\right]^{\mathbf{T}}\left[\mathbf{R}_{1 \mathbf{C}}\right]\right] \overrightarrow{\mathbf{X}}_{1}+\frac{\sigma_{1 \mathbf{C}}}{\sigma_{2 \mathbf{C}}}\left[\mathbf{R}_{2 \mathbf{C}}\right]^{\mathbf{T}}\left[\mathbf{R}_{1 \mathbf{C}}\right] \overrightarrow{\mathbf{V}}_{1}+\frac{\left[\mathbf{R}_{2 \mathbf{C}}\right]^{\mathbf{T}}}{\sigma_{2 \mathbf{C}}} \\
& \times\left(\dot{\mathbf{T}}_{1 \mathbf{C}}-\dot{\mathbf{T}}_{2 \mathbf{C}}\right)-\left(\frac{\dot{\sigma}_{2 \mathbf{C}}}{\sigma_{2 \mathbf{C}}}+\left[\mathbf{R}_{2 \mathbf{C}}\right]^{\mathbf{T}}\left[\dot{\mathbf{R}}_{2 \mathbf{C}}\right]\right) \frac{\left[\mathbf{R}_{2 \mathbf{C}}\right]^{\mathbf{T}}}{\sigma_{2 \mathbf{C}}}\left(\overrightarrow{\mathbf{T}}_{1 \mathbf{C}}-\overrightarrow{\mathbf{T}}_{2 \mathbf{C}}\right)
\end{aligned}
$$

For a general n-solution case, there exist $\mathbf{C}_{2}^{\mathbf{n}}=\mathbf{n}(\mathbf{n}-1) / 2$ transformations between them. However, one can prove that only $(\mathbf{n}-1)$ sets of transformation parameters are independent. The rest of the $\mathbf{n}(\mathbf{n}-1) / 2-(\mathbf{n}-1)=(\mathbf{n}-1)(\mathbf{n}-2) / 2$ sets of transformation parameters can be analytically derived from these $(\mathbf{n}-1)$ independent sets of parameters. As a result, the parameter estimates could become highly or partially dependent, if these dependent transformations share common observables. In such a case, further treatment on the common observables becomes necessary, as will be discussed in the following section.

Table 2. Summary of Input SINEX Files

Table 1. Information Used to Determine Transformation Parameters in Three-Solution Combination

\begin{tabular}{lcc}
\hline Transformation & $\begin{array}{c}\text { All possible } \\
\text { information }\end{array}$ & $\begin{array}{c}\text { Independent } \\
\text { information }\end{array}$ \\
\hline $\mathbf{X}_{1} \rightarrow \mathbf{X}_{\mathbf{C}}$ & $\left(X_{1} \cap X_{C}\right)=\mathrm{I}+\mathrm{II}$ & $\left(X_{1} \cap X_{C}\right)=\mathrm{I}+\mathrm{II}$ \\
$\mathbf{X}_{2} \rightarrow \mathbf{X}_{\mathbf{C}}$ & $\left(X_{2} \cap X_{C}\right)=\mathrm{I}+\mathrm{III}$ & $\left(X_{2} \cap X_{C}\right)=\mathrm{I}+\mathrm{III}$ \\
$\mathbf{X}_{1} \rightarrow \mathbf{X}_{2}$ & $\left(X_{1} \cap X_{2}\right)=\mathrm{I}+\mathrm{IV}$ & $\left(X_{1} \cap X_{2}\right)-\left(X_{1} \cap X_{2} \cap X_{C}\right)=\mathrm{IV}$ \\
\hline
\end{tabular}

\begin{tabular}{lccc}
\hline Solution ID & SINEX1 & SINEX2 & SINEX3 \\
\hline SINEX name & ngs94-03.snx & IGS03P33_RS99.snx & IGS05P15.snx \\
Number of records $^{\mathrm{a}}$ & 292 & 99 & 277 \\
Number of sites $^{\text {Reference epoch }}$ & 251 & 99 & 247 \\
Ref & $1,998.0$ & $1,998.0$ & $1,998.0$ \\
\hline
\end{tabular}

${ }^{\mathrm{a}}$ Including multiple records at the same site. 

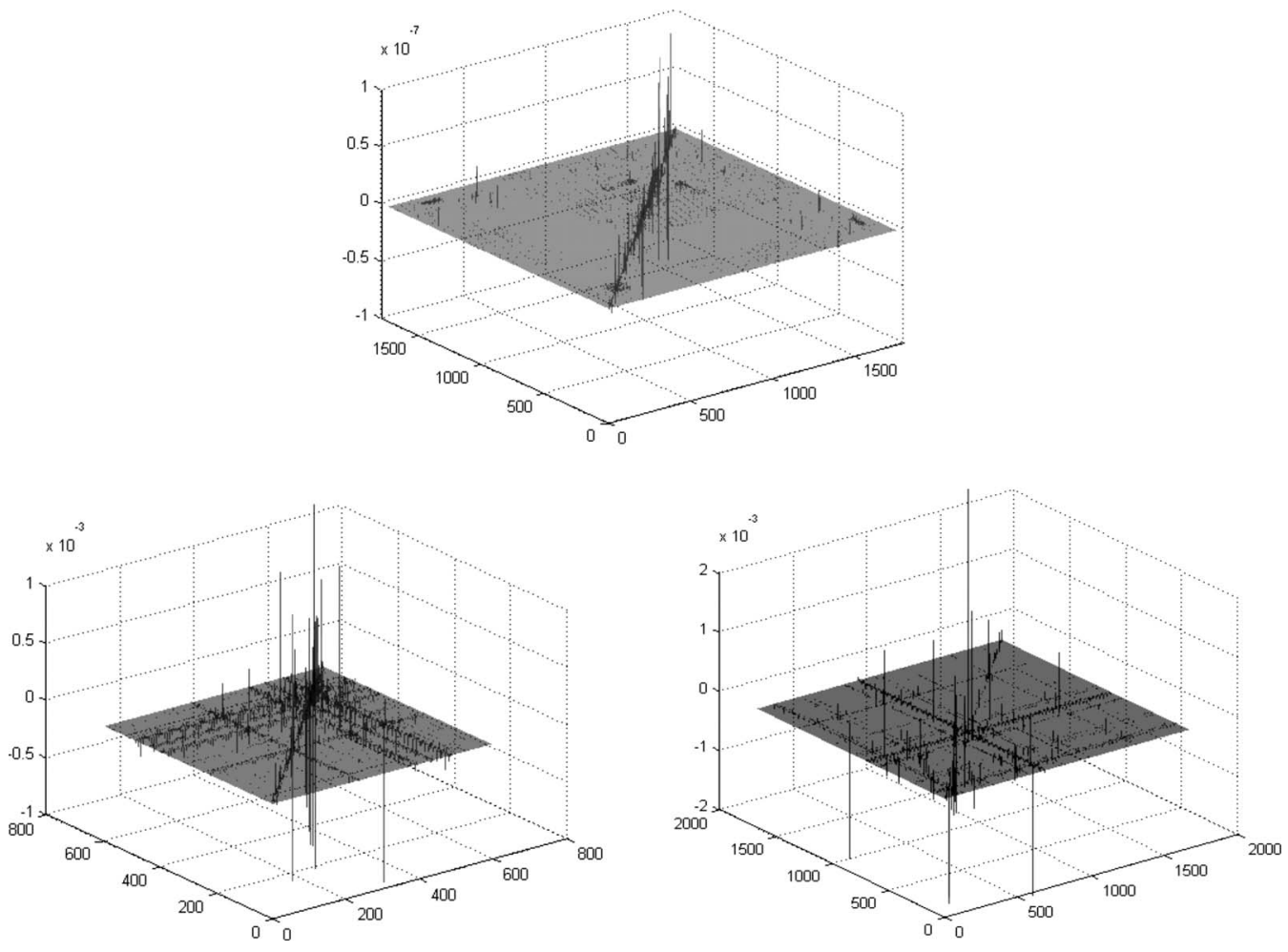

Fig. 4. Three-dimensional mesh representations of input covariance matrices (upper: ngs94-03.snx; lower left: IGS03P33_RS99.snx; lower right: IGS05P15.snx)

\section{Observable Dependency Analysis}

As shown in the previous section, there are dependent transformations between multiple solutions and problems could occur when common observables (i.e., station coordinates and velocities) are used to estimate the dependent sets of transformation parameters. To avoid this problem, all observables should be carefully arranged so that any set of observables is only used for estimating independent parameters. A Venn diagram in Fig. 2 shows the information (i.e., observables) involved in a combination of three different solutions. While estimating the transformation $\left(\mathbf{X}_{\mathbf{1}} \rightarrow \mathbf{X}_{\mathbf{C}}\right)$, the common information in Regions I and II is used. Similarly, the common information in Regions I and III is used to determine the transformation $\left(\mathbf{X}_{2} \rightarrow \mathbf{X}_{\mathbf{C}}\right)$. As a result, the information in Region I is used twice. This does not cause any problem since the same information is used in estimating two independent sets of transformation parameters. However, while forming the condition equations between $\mathbf{X}_{1}$ and $\mathbf{X}_{2}$, the information in Region I should not be used again due to the fact that the transformation $\left(\mathbf{X}_{1} \rightarrow \mathbf{X}_{2}\right)$ is dependent on the transformation $\left(\mathbf{X}_{1} \rightarrow \mathbf{X}_{\mathbf{C}}\right)$ and the transformation $\left(\mathbf{X}_{2} \rightarrow \mathbf{X}_{\mathbf{C}}\right)$, as discussed in the previous section. In other words, if the same information is being used more than once in determining dependent parameter values, an overestimation problem will be the result. In this case, only the information in Region IV should be used to construct condition equations between $\mathbf{X}_{1}$ and $\mathbf{X}_{2}$ using Eq. (6).

Table 1 summarizes the appropriate sets of observables that should be used in determining the transformations between three given terrestrial solutions.

\section{Case Study}

In this study, the real data of three globally distributed network solutions, one provided by the National Geodetic Survey (NGS), NOAA, and the other two by the IGS, are combined using the proposed combination approach. These solutions are expressed in the Software INdependent EXchange (SINEX) format. Fig. 3 and Table 2 show the site distributions and a summary of these three network solutions. As shown in Table 2, there are multiple records at the same site in the SINEX files, which typically come from a discrete change of antenna positions at the same sites. Besides, the second and third SINEX files (i.e., IGS03P33_RS99.snx and IGS05P15.snx) are IGS solutions obtained at different epochs, but both are transformed and given at the same epoch (1998.0). It is also noted that the covariance matrix provided in the first SINEX file (i.e., by NGS) is several orders of magnitude smaller than the other two SINEX files. Apparently, these covariance values do not give a direct indication for the quality of station coordinates, but for the statistical structure (i.e., correlations) between all stations in a network solution. See Fig. 4 for a graphic representation of these covariance matrices.

These three solutions are combined using the Geodetic Network Analysis Tool (GNAT) software that has been developed to implement the combination approach proposed by this research. After carrying out the observable dependency analysis (ODA) procedure, a Venn diagram is generated as shown in Fig. 5. The ODA result is then used to locate appropriate sets of observables while estimating the transformation parameters. Table 3 lists the estimated parameter values. Note that the first SINEX is used as 


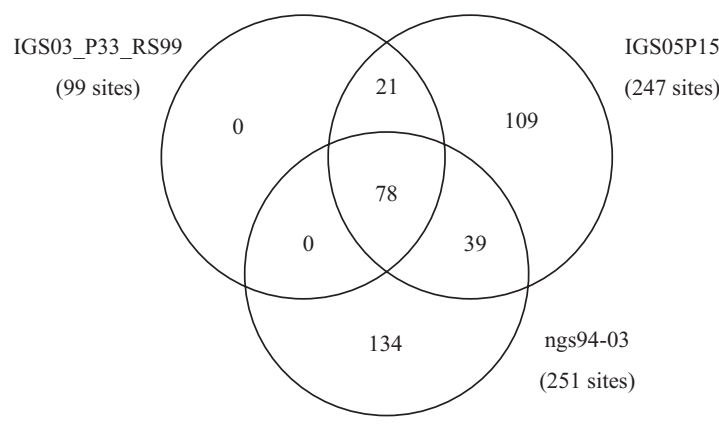

Fig. 5. Venn diagram showing numbers of sites in each region between three SINEX solutions

the reference solution in this study. A direct application of reference frame transformation converts the second and third solutions into the reference frame defined by this reference solution. A combined solution that contains a union of all sites from three input solutions but expressed in a common reference frame is obtained.

Since the complete geometric relations between solutions are taken into account, the combination solution should be unique and self-consistent. In this case study, the 21 common sites in Region IV (intersection of SINEX 2 and SINEX3) are examined. Their positional coordinates in the combination solution are computed by: (1) using the coordinates in SINEX2 and applying transformation parameters $\mathbf{P}_{2 \mathbf{C}}$ (i.e., $\overrightarrow{\mathbf{X}}_{2 \mathbf{C}}^{\mathbf{I V}}=\sigma_{2 \mathbf{C}}\left[\mathbf{R}_{2 \mathbf{C}}\right] \overrightarrow{\mathbf{X}}_{2}^{\mathbf{I V}}+\overrightarrow{\mathbf{T}}_{2 \mathbf{C}}$ ); and (2) using the coordinates in SINEX3 and applying transformation parameter $\mathbf{P}_{3 \mathbf{C}}$ (i.e., $\quad \overrightarrow{\mathbf{X}}_{3 \mathbf{C}}^{\mathbf{I V}}=\sigma_{3 \mathbf{C}}\left[\mathbf{R}_{3 \mathbf{C}}\right] \overrightarrow{\mathbf{X}}_{3}^{\mathbf{I V}}+\overrightarrow{\mathbf{T}}_{3 \mathbf{C}}$ ). It is confirmed that these two computations result in an identical solution (i.e., $\left|\overrightarrow{\mathbf{X}}_{3 \mathbf{C}}^{\mathbf{I V}}-\overrightarrow{\mathbf{X}}_{2 \mathbf{C}}^{\mathbf{I V}}\right|<10^{-9} \mathrm{~m}$ ). However, if the proposed approach is not considered but a direct transformation of SINEX 2 and SINEX3 is simply applied, those 21 common sites will have inconsistent values in the combined solution. In this study, the positional inconsistency for these 21 sites reaches the $10^{-3}-10^{-2} \mathrm{~m} \quad$ level (i.e., $\quad$ mean $\left|\overrightarrow{\mathbf{X}}_{3 \mathbf{C}}^{\mathbf{I V}}-\overrightarrow{\mathbf{X}}_{2 \mathbf{C}}^{\mathbf{I V}}\right|=0.006 \mathrm{~m}$; $\max \left|\overrightarrow{\mathbf{X}}_{3 \mathbf{C}}^{\mathbf{I V}}-\overrightarrow{\mathbf{X}}_{2 \mathbf{C}}^{\mathbf{I V}}\right|=0.060 \mathrm{~m}$ ), which would pose a significant problem due to the neglect of complete geometric relations between multiple solutions.

\section{Remarks}

The main purpose of a combination is to produce an integrated solution that is based on a consistent reference frame definition. To achieve this goal, the complete geometric relations (and interrelations) should be fully taken into consideration. By applying the proposed combination approach, one is able to identify appropriate geometry and resolve dependencies between observables and parameters, and thus obtain a unique and self-consistent combination solution. On the other hand, if the proposed approach is not implemented, errors could occur, deteriorating the quality of the combination solution.

It should be emphasized that this research is focused on the geometric combination of multiple solutions based on a similarity transformation model. Although it is possible to combine time series solutions under the proposed model, it is not recommended to do so. The reason is that often there is not necessarily a specific geometric relationship between the various time series due to possible discrete movements (i.e., noncommon motions) at some sites caused by earthquakes, antenna changes, etc. A better way to combine time series solutions is to apply the Kalman filter technique, which allows one to estimate/predict the solution at a specific epoch based on the observations during a period of time (see, e.g., Dong et al. 1998; and Herring et al. 2006).

\section{Acknowledgments}

This research was supported by the National Geodetic Survey, NOAA, under Contract No. NCNL2000-6-00004. Comments by three anonymous reviewers are also gratefully acknowledged.

Table 3. Parameter Estimates for Three-Solution Combination

\begin{tabular}{|c|c|c|c|}
\hline & $\begin{array}{c}\text { SINEX } 2 \rightarrow \text { SINEX } 1 \\
\mathbf{P}_{2 \mathbf{C}} \\
\end{array}$ & $\begin{array}{c}\text { SINEX } 3 \rightarrow \text { SINEX } 1 \\
\mathbf{P}_{3 \mathbf{C}} \\
\end{array}$ & $\begin{array}{c}\text { SINEX } 2 \rightarrow \text { SINEX3 } \\
\mathbf{P}_{23}=\mathbf{P}_{2 \mathbf{C}}-\mathbf{P}_{3 \mathbf{C}}{ }^{b}\end{array}$ \\
\hline$\sigma(\mathrm{ppb})^{\mathrm{a}}$ & $-0.12 \pm 0.07$ & $-0.19 \pm 0.01$ & $0.07 \pm 0.07$ \\
\hline$r x{(\text { mas })^{c}}^{c}$ & $0.00 \pm 0.01$ & $-0.07 \pm 0.01$ & $0.07 \pm 0.02$ \\
\hline$r y$ (mas) & $-0.06 \pm 0.01$ & $-0.05 \pm 0.01$ & $-0.02 \pm 0.01$ \\
\hline$r z$ (mas) & $-0.06 \pm 0.01$ & $-0.05 \pm 0.01$ & $-0.01 \pm 0.01$ \\
\hline$T x(\mathrm{~cm})$ & $0.00 \pm 0.03$ & $0.22 \pm 0.06$ & $-0.22 \pm 0.06$ \\
\hline$T y(\mathrm{~cm})$ & $0.00 \pm 0.04$ & $0.06 \pm 0.08$ & $-0.06 \pm 0.08$ \\
\hline$T z(\mathrm{~cm})$ & $-0.22 \pm 0.03$ & $-0.55 \pm 0.05$ & $0.33 \pm 0.05$ \\
\hline$\sigma \_d(\mathrm{ppb} /$ year $)$ & $-0.26 \pm 0.03$ & $-0.27 \pm 0.01$ & $0.01 \pm 0.03$ \\
\hline$r x \_d$ (mas/year) & $0.01 \pm 0.00$ & $0.01 \pm 0.00$ & $0.00 \pm 0.01$ \\
\hline$r y \_d$ (mas/year) & $0.00 \pm 0.00$ & $-0.01 \pm 0.00$ & $0.01 \pm 0.01$ \\
\hline$r z, d$ (mas/year) & $0.00 \pm 0.00$ & $0.01 \pm 0.00$ & $-0.01 \pm 0.01$ \\
\hline$T x_{-} d(\mathrm{~cm} /$ year $)$ & $0.07 \pm 0.01$ & $0.04 \pm 0.02$ & $0.03 \pm 0.02$ \\
\hline$T y \_d(\mathrm{~cm} /$ year $)$ & $-0.22 \pm 0.02$ & $-0.24 \pm 0.01$ & $0.02 \pm 0.02$ \\
\hline$T z \_d(\mathrm{~cm} /$ year $)$ & $-0.16 \pm 0.01$ & $-0.15 \pm 0.01$ & $-0.01 \pm 0.01$ \\
\hline
\end{tabular}

${ }^{\mathrm{a} p \mathrm{pb}}=10^{-9}$.

${ }^{b}$ Derived values based on Eqs. (7)-(12), which in this case can be approximated by $\mathbf{P}_{23}=\mathbf{P}_{2 \mathbf{C}}-\mathbf{P}_{3 \mathbf{C}}$.

${ }^{c}$ mas $=$ milli-arc-second. 


\section{References}

Altamimi, Z., and Boucher, C. (2003). "Multi-technique combination of time series of station positions and Earth orientation parameters." Proc., IERS Workshop on Combination Research and Global Geophysical Fluids, IERS Tech. Note No. 30, B. Richter, W. Schwegmann, and W. R. Dick, eds., Verlag des Bundesamts für Kartographie und Geodäsie, Frankfurt am Main, Germany, 102-106.

Altamimi, Z., Sillard, P., and Boucher, C. (2002), "ITRF2000: A new release of the international terrestrial reference frame for Earth Science applications." J. Geophys. Res., 107(B10), ETG2/1-19.

Boucher, C., and Altamimi, Z. (1989). "The initial IERS Terrestrial Reference Frame." IERS Tech. Note No. 1, Observatoire de Paris, Paris.

Boucher, C., and Altamimi, Z. (1992). "The EUREF terrestrial reference system and its first realizations." Tech. Rep. No. 52, Veröffentlichungen der Bayerischen Kommissionen für die Internationalen Erdmessung, München, Germany.

Boucher, C., and Altamimi, Z. (2001). "ITRS, PZ90 and WGS84: Current realizations and the related transformation parameters." J. Geodesy, Berlin, 75(11), 613-619.

DeMets, C., Gordon, R., Argus, D., and Stein, S. (1990). "Current plate motions." Geophys. J. Int., 101(2), 425-478.

Dong, D., Herring, T. A., and King, R. W. (1998). "Estimating regional deformation from a combination of space and terrestrial geodetic data." J. Geodesy, Berlin, 72(4), 200-214.

Ferland, R. (2004). "Reference frame working group technical report." International GPS Service 2001-2002 Technical Rep. IGS Central Bureau, Jet Propulsion Laboratory, California Institute of Technology, Pasadena, Calif.

Grafarend, E. (1984). "Variance-covariance-component estimation of
Helmert type in the Gauß-Helmert model." ZFV. Z. Vermessungswes., 109, 34-44.

Han, J. Y., and van Gelder, B. H. W. (2006). "Stepwise parameter estimations in a time-variant similarity transformation." J. Surv. Eng., 132(4), 141-148.

Herring, T. A., King, R. W., and McClusky, S. C. (2006). GLOBK reference manual: Global Kaman filter VLBI and GPS analysis program, Release 10.3, Dept. of Earth, Atmospheric, and Planetary Sciences, Massachusetts Institute of Technology, Cambridge, Mass.

Ray, J., et al. (1999). "IERS working group on ITRF datum. Final report." International Earth Rotation Service, Paris, 〈http:// hpiers.obspm.fr/iers/itrf/ITRF-WG.Report) (Aug. 2008).

Ray, J., and Altamimi, Z. (2005). "Evaluation of co-location ties relating the VLBI and GPS reference frames." J. Geodesy, Berlin, 79(4-5), 189-195.

Sahin, M., Cross, P. A., and Sellers, P. C. (1992). "Variance component estimation to satellite laser ranging." Bull. Geod., 66(3), 284-295.

Schwarz, C. R. (1989). "North American datum of 1983." NOAA Professional Paper No. NOS2, U.S. Dept. of Commerce, National Oceanic and Atmospheric Administration, Silver Spring, Md.

Snay, R. A. (1999). "Using the HTDP software to transform spatial coordinates across time and between reference frames." Surv. Land Inf. Sys., 59(1), 15-25.

Soler, T. (1998). "A compendium of transformation formulas useful in GPS work." J. Geodesy, Berlin, 72(7-8), 482-490.

Soler, T., and Snay, R. A. (2004). "Transforming positions and velocities between the international terrestrial reference frame of 2000 and North American datum of 1983." J. Surv. Eng., 130(2), 49-55.

Steed, J. B. (1995). "Geocentric datum of Australia." Surv. World, 4(1), $14-17$. 